\title{
REVIEW
}

\section{Molecular mechanisms in the formation of the medial longitudinal fascicle}

\author{
Mansoor Ahsan, Kerry-lyn Riley and Frank R. Schubert \\ Institute of Biomedical and Biomolecular Sciences, School of Biological Sciences, University of Portsmouth, UK
}

\begin{abstract}
The first neurons in the vertebrate brain form a stereotypical array of longitudinal and transversal axon tracts, the early axon scaffold. This scaffold is thought to lay down the basic structure for the later, more complex neuronal pathways in the brain. The ventral longitudinal tract is pioneered by neurons located at the ventral midbrainforebrain boundary, which form the medial longitudinal fascicle. Recent studies have shed some light on the molecular mechanisms that control the development of the medial longitudinal fascicle. Here, we show that patterning molecules, notably the ventralizing signalling molecule Shh, are involved in the formation of medial longitudinal fascicle neurons and in medial longitudinal fascicle axon guidance. Downstream of Shh, several homeobox genes are expressed in the tegmentum. We describe the expression patterns of Sax1,Emx2, Six3, Nkx2.2 and Pax6 in the mesencephalon and pretectum in detail. Furthermore, we review the evidence of their molecular interactions, and their involvement in neuronal fate specification. In particular, Sax1 plays a major role in fate determination of medial longitudinal fascicle neurons. Finally, we discuss the available data on axon guidance mechanisms for the medial longitudinal fascicle, which suggest that different guidance molecules such as class 3 Semaphorins, Slits and Netrins act to determine the caudal and ventral course of the medial longitudinal fascicle axons.
\end{abstract}

Key words early axon scaffold; Emx2; medial longitudinal fascicle; mesencephalon; pretectum; Sax1; Shh.

\section{Introduction}

During early embryogenesis, the basic organization of the mature vertebrate brain is outlined by pioneering axons that form a well-conserved set of axon tracts, the early axon scaffold (reviewed in Nieuwenhuys, 1998). This axon scaffold was first described in zebrafish (Chitnis \& Kuwada, 1990; Wilson et al. 1990), and has since been studied in a variety of non-amniote (e.g. Hartenstein, 1993; Doldan et al. 2000) and amniote species (e.g. Easter et al. 1993; Chedotal et al. 1995; Mastick \& Easter, 1996). In fish and amphibians (Fig. 1A; reviewed in Hjorth \& Key, 2002), it comprises two longitudinal tracts (tract of the postoptic commissure $=$ TPOC, medial longitudinal fascicle $=$ MLF) and three transversal tracts (supraoptic tract $=$ SOT, dorsoventral diencephalic tract = DVDT, tract of the posterior commissure $=$ TPC). The early axon scaffold also includes four commissures (anterior commissure $=A C$, postoptic

\section{Correspondence}

Dr Frank R. Schubert, Institute of Biomedical and Biomolecular

Sciences, School of Biological Sciences, University of Portsmouth,

King Henry Building, Portsmouth PO1 2DY, UK.

E: frank.schubert@port.ac.uk

The first two authors contributed equally to the study.

Accepted for publication 8 June 2007 commissure $=\mathrm{POC}$, posterior commissure $=\mathrm{PC}$, ventral commissure $=\mathrm{VC}$ ). The cell bodies giving rise to these tracts are largely clustered in three areas, the telencephalon (dorso-rostral cluster), hypothalamus (ventro-rostral cluster) and tegmentum (ventro-caudal cluster). A similar arrangement, particularly evident in the longitudinal tracts, is found in amniotes (Fig. 1B; reviewed in Nieuwenhuys, 1998). Neurons in the hypothalamus project axons into the medial bundle to form the TPOC and mammillo-tegmental tract (= MTT), while the MLF is formed by neurons located in the tegmentum of pretectum and midbrain. In contrast, most transversal tracts are not readily identifiable in early amniote embryos. A notable exception is the TPC, formed by neurons at the midbrain-forebrain boundary (MFB), which is a prominent landmark of the pretectum. Also, a projection from the olfactory region (e.g. Windle \& Austin, 1936) may be the equivalent of the SOT, while the $A C$ and POC only form at later embryonic stages. Interestingly, the neurons of the mesencephalic nucleus of the trigeminal nerve differentiate early, thus establishing an early tract in the dorsal mesencephalon (DTmesV) that initiates the lateral longitudinal tract. Furthermore, the somatic motor neurons of the oculomotor and trochlear nuclei form distinct cell masses in the early tegmentum.

Among the tracts of the early axon scaffold, the MLF is particularly prominent. It is conserved in all vertebrate 


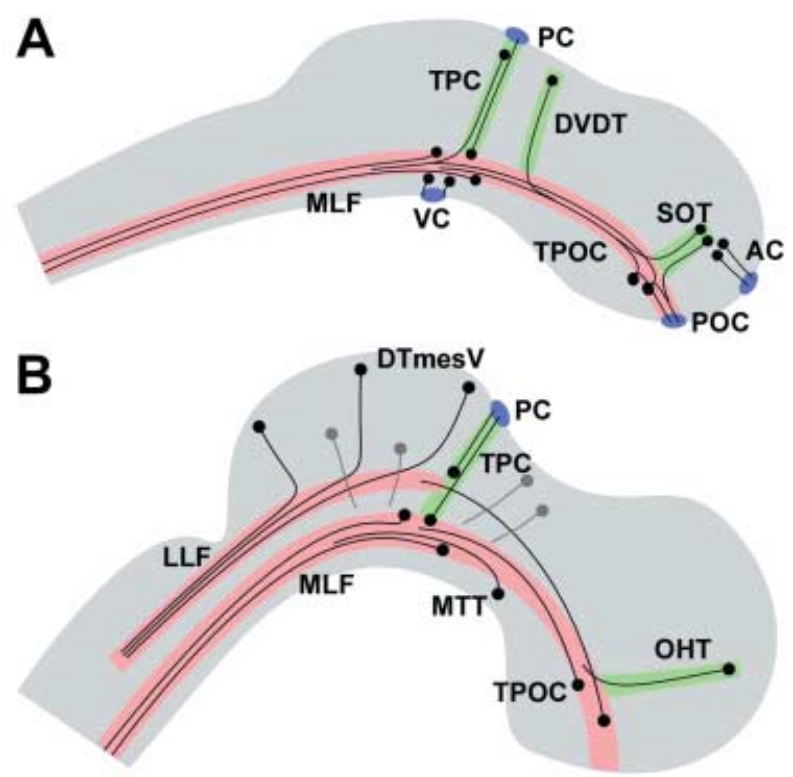

Fig. 1 The early axon scaffold. Schematic representation of early axon tracts in an idealized non-amniote (A) and amniote (B) embryo. Longitudinal bundles are outlined in red, transversal tracts in green. Commissures are depicted as blue ovals. A prominent structure in the brain of all vertebrate embryos is the medial longitudinal bundle to which the TPOC, MTT and MLF contribute. In amniotes, the axons of the DTmes $V$ establish the lateral longitudinal bundle already in the early brain (in chick visible at $\mathrm{HH} 14$ ), while in non-amniotes this bundle develops later. In contrast, several distinct transversal tracts (TPC, DVDT, SOT) and commissures (AC, POC, PC, VC) appear early in the brain of fishes and amphibians, while in mouse and chick, only the TPC with its associated commissure is prominent in the early brain. A possible homologue of the SOT is formed by axons growing from the olfactory region towards the ventral forebrain (OHT) in amniotes. In addition, several other neurons in forebrain and midbrain extend their axons transversally, but these do not form distinct tracts at early stages of development. AC, anterior commissure; DTmesV, dorsal tract of the mesencephalic nucleus of the trigeminus; DVDT, dorsoventral diencephalic tract; LLF, lateral longitudinal fascicle; MLF, medial longitudinal fascicle; MTT, mamillo-tegmental tract; OHT, olfactory-hypothalamic tract; PC, posterior commissure; POC, postoptic commissure; SOT, supraoptic tract; TPC, tract of the posterior commissure; TPOC, tract of the postoptic commissure; VC, ventral commissure.

species, and several studies have highlighted the MLF as one of the earliest tracts in the brain, pioneering the medial longitudinal axon bundle (e.g. McConnell \& Sechrist, 1980; Puelles et al. 1987; Ross et al. 1992). The MLF originates from neurons located at the ventral MFB, both in the mesencephalon and in the pretectum (Fig. 2A,C). This region is marked by the expression of Gap43 (Fig. 2B), a gene implicated in active axon outgrowth (e.g. Skene \& Willard, 1981). One group of MLF neurons, predominantly located in the mesencephalon, are scattered over a wide area dorsal to the MLF. They project their axons first ventrally, before turning caudally into the MLF (Fig. 2A,C). In contrast, most pretectal MLF neurons are located directly in the path of the MLF axons, are densely packed and project their axons immediately caudally (Fig. 2A,C,D). At later stages, the pretectal MLF neurons are intermingled with neurons projecting into the TPC (Fig. 2D). It is unclear whether the mesencephalic and pretectal parts of the nucleus of the MLF (nMLF) are distinct. Puelles et al. (1987) distinguish between the pretectal area nucleus fasciculi longitudinalis medialis and the mesencephalic area tegmentalis, while other authors collectively refer to these as the ventro-caudal cluster (e.g. Hjorth \& Key, 2002).

Despite the prominence of the MLF in the early brain, little is known about the formation of this tract, particularly the specification of its neurons, and the factors guiding the outgrowth of its axons. In this article, we will review the current knowledge of the molecular mechanisms controlling MLF formation, and will present novel data on patterning, neuronal specification and axon guidance events in MLF development.

\section{Materials and methods}

\section{Chick embryos}

Fertilized domestic chicken eggs were obtained from Henry Stewart \& Co. Ltd (Peterborough, UK). They were incubated at $38{ }^{\circ} \mathrm{C}$ for the required stage. Embryos were prepared in phosphate-buffered saline (PBS; Fisher Scientific) and fixed in $4 \%$ paraformaldehyde, made up in PBS, for at least $24 \mathrm{~h}$. Staging was according to Hamburger \& Hamilton (1951) criteria.

\section{Expression analysis}

We employed RNA probes for chick Sax1 (Spann et al. 1994), Emx2 (Bell et al. 2001), Is/1 (Tsuchida et al. 1994), Nkx2.2 (F. R. Schubert, unpublished data), Pax6 (Goulding et al. 1993) and Six3 (Chapman et al. 2002) for our analysis.

Formaldehyde-fixed chick embryos were bleached in $6 \% \mathrm{H}_{2} \mathrm{O}_{2} /$ methanol, and stored in methanol at $-20^{\circ} \mathrm{C}$. After rehydration in PBS, embryos were treated three times for 20 min with detergent mix (1\% IGEPAL, 1\% SDS, $0.5 \%$ dexoycholate, $50 \mathrm{~mm}$ Tris-HCl, pH 8, 1 mm EDTA, $0.15 \mathrm{~m}$ $\mathrm{NaCl}$ ) and then briefly re-fixed in $4 \%$ paraformaldehyde. Prehybridization was for $1 \mathrm{~h}$ at $65{ }^{\circ} \mathrm{C}$ in Prehyb mix $(50 \%$ formamide, $5 \times \mathrm{SSC}, \mathrm{pH} 4.5,2 \% \mathrm{SDS}, 2 \%$ Boehringer blocking reagent, $250 \mu \mathrm{g} \mathrm{mL}^{-1} \mathrm{RNA}, 100 \mu \mathrm{g} \mathrm{mL}^{-1}$ heparin, in $\mathrm{H}_{2} \mathrm{O}$ ). Embryos were hybridized overnight in Prehyb mix with $1 \mu \mathrm{g} \mathrm{mL}^{-1}$ of labelled probe. After several washes in Solution X ( $50 \%$ formamide, $2 \times \mathrm{SSC}, \mathrm{pH} 4.5,1 \% \mathrm{SDS})$ at $65^{\circ} \mathrm{C}$, embryos were transferred to MABT $(0.1 \mathrm{~m}$ maleic acid, $0.15 \mathrm{M}$ $\mathrm{NaCl}, 0.1 \%$ Tween 20). Non-specific antibody binding was blocked by incubating the embryos in $20 \%$ goat serum, $2 \%$ Boehringer blocking reagent in MABT, followed by binding to the alkaline phosphatase-conjugated antibody (Roche). In double labelling experiments, digoxigenin- and fluoresceinlabelled probes were consecutively detected with the 


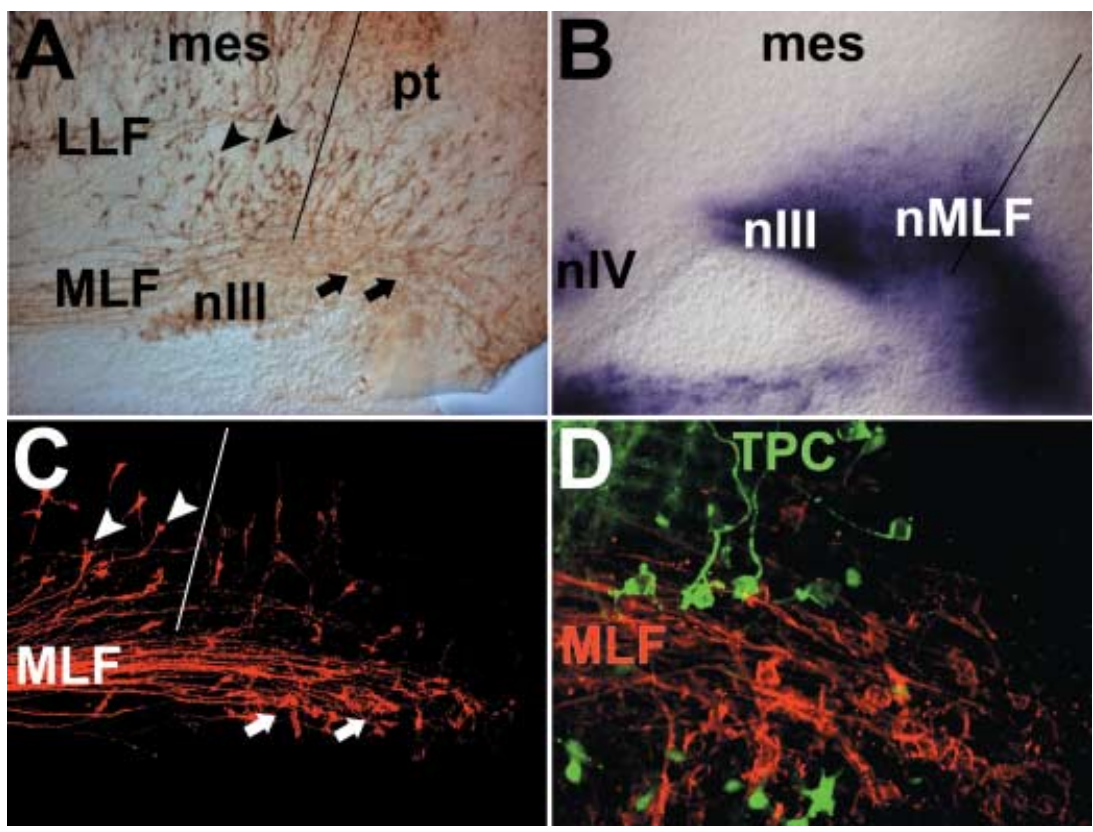

Fig. 2 The medial longitudinal fascicle in the chick embryo. (A) The ventral midbrain-forebrain border region of an HH18 chick embryo, immuno-labelled for Neurofilament-M. The medial (MLF) and lateral (LLF) longitudinal axon bundles are traversing the midbrain. The MLF neurons are located in two main areas, firstly loosely scattered in the rostral mesencephalon, dorsal to the course of the MLF (arrowheads), secondly intermingled with the MLF axons in the ventral pretectum (arrows). (B) The nucleus of the MLF, along with the oculomotor and trochlear nuclei, expresses Gap43 at $\mathrm{HH} 18$. (C) Retrograde labelling of the MLF in an HH18 embryo (from rhombomere 1/2) again reveals the two populations of MLF neurons in the mesencephalon (arrowheads) and pretectum (arrows). Note the absence of labelling rostral to the pretectum. (D) At HH21, neurons contributing to the TPC (green, labelled from the dorsal pretectum) are intermingled with MLF neurons (red, labelled from rhombomere 1/2) in the ventral pretectum. LLF, lateral longitudinal fascicle; mes, mesencephalon; MLF, medial longitudinal fascicle; nIII, oculomotor nucleus; nIV, trochlear nucleus; nMLF, nucleus of the MLF; pt, pretectum; TPC, tract of the posterior commissure.

respective antibody. After washing in $M A B T$, the bound antibody was detected using NBT/BCIP (Roche) and Vector Red (Vector Laboratories) as blue and red substrates, respectively.

\section{Retrograde labelling of MLF and PC}

Specific axon tracts in the embryonic brain were retrogradelabelled with lipophilic dyes (Dil, DiO; Molecular Probes). The mesenchyme was removed from chick embryos, and isolated, hemisected brains were fixed flat on black nitrocellulose membrane (Schleicher \& Schuell). Crystalline Dil was applied to the ventral part of rhombomere $1 / 2$ to label the MLF, while DiO was used to label the PC from the roof plate of the caudal pretectum.

To analyse the expression of Sax1 in relation to MLF neurons, we retrogradely labelled the MLF from the rostral hindbrain by fluorescein-labelled dextran (Molecular Probes). After the in situ hybridization for Sax1, the fluorescein label was detected with an alkaline phosphatase-conjugated antifluorescein antibody (Roche), using Vector Red as substrate.

\section{Immunohistochemistry}

Neurons and axon tracts in the embryonic chick brains were detected with an antibody against neurofilament-M
(Zymed RMO270), visualized by a peroxidase-conjugated anti-mouse antibody (Jackson Laboratories) using diaminobenzidine (Vector Laboratories) as substrate. When combined with mRNA detection, the primary antineurofilament antibody was applied after the wholemount in situ hybridization procedure.

\section{Electroporation}

The pXeX-Shh construct (Agarwala et al. 2001) was a gift from C. Ragsdale. The pCA $\beta$-Sax 1 construct has been described elsewhere (Schubert \& Lumsden, 2005). Expression constructs were used at a concentration of $1 \mu \mathrm{g} \mu \mathrm{L}^{-1}$, with Fast Green added to $0.1 \%$ to facilitate visualization of the DNA solution. The DNA solution was injected into the rostral neural tube of Hamburger \& Hamilton $(\mathrm{HH})$ stages 11-13 chick embryos, using a PV820 Picopump (World Precision Instruments). The platinum anode $(0.25 \mathrm{~mm}$ in diameter) was placed lateral to the midbrain, while the flame-sharpened tungsten cathode $(0.125 \mathrm{~mm}$ in diameter before sharpening) was inserted into the neural tube. Two pulses of $12.5 \mathrm{~V} /$ $50 \mathrm{~ms}$ were applied, using an Ovodyne electroporator (Intracel). The eggs were then incubated for a further 1-3 days. 


\section{Sectioning and photography}

Where required, embryos were sectioned after the wholemount in situ hybridization or immunohistochemistry procedures. Sections of $30 \mu \mathrm{m}$ were cut on a vibratome (Pelco), and mounted in $80 \%$ glycerol in PBS.

Digital photographs of whole embryos, dissected brain tissue or sections were taken using differential interference contrast, either in a Zeiss Axiophot or a Nikon Eclipse microscope. Subsequent processing and assembly of the images was performed with Adobe Photoshop CS.

Fluorescent samples were analysed in an Olympus Fluoview or Zeiss LSM510 confocal microscope, combining several frames from a Z-stack into single images.

\section{Setting the scene: secondary organizers influence the early axon scaffold}

The first MLF neurons differentiate shortly after the initial patterning of the brain, in zebrafish $16 \mathrm{~h}$ after fertilization (Ross et al. 1992), and in the chick at HH13 (e.g. McConnell \& Sechrist, 1980; Puelles et al. 1987). They are positioned at the ventral MFB, a region under the influence of the ventralizing factor Shh expressed in the floor plate, and the caudalizing factor Fgf8 secreted from the isthmic organizer. The roles of both signalling systems in early brain patterning have been studied in detail, although not specifically in relation to the development of the MLF.

Fgf8 is the key signalling molecule secreted from the isthmic organizer, and functions in the initial induction of the mesencephalon, and the subsequent establishment of rostrocaudal polarity across the midbrain (reviewed in Nakamura \& Watanabe, 2005). Apart from the isthmus, Fgf8 is also expressed in the anterior neural plate where it is involved in the formation of the telencephalon. Mutations affecting the Fgf8 signalling pathway or the establishment of the signalling centres result in patterning defects and alterations to the early axon scaffold. In the zebrafish mutants ace (= Fgf8, Shanmugalingam et al. 2000) and noi (= Pax2, Macdonald et al. 1997), the formation of the AC and POC is disrupted, which is attributed to erroneous development of midline glia cells.

Shh is expressed in the notochord and the floor plate along the whole rostrocaudal axis, and at later stages in the zona limitans intrathalamica (ZLI). In the spinal cord, Shh acts as a morphogen to specify the fate of different neuronal types in the basal plate (Briscoe et al. 2000). Shh is likewise involved in dorsoventral patterning of the brain. In the midbrain, Shh has been suggested to suppress the development of the tectum and to promote the formation of the tegmentum (Watanabe \& Nakamura, 2000), because ectopic expression of Shh in the dorsal mesencephalon induces the expression of ventral genes (Watanabe \& Nakamura, 2000; Agarwala et al. 2001). An additional later function of Shh contributes to the rostrocaudal patterning of the diencephalon by the ZLI (Kiecker \& Lumsden, 2004). Mutations in the Shh pathway also affect the early axon scaffold. In the zebrafish smu (= smoothened) mutant, pathfinding defects of rostral tracts have been attributed to erroneous expression of axon guidance molecules in the ventral midline (Varga et al. 2001). The effect of Shh on the MLF has not been studied. However, in smu zebrafish the expression of the homeobox gene Six3, a marker for the ventrodaucal cluster at the MFB, is reduced (Tallafuss et al. 2003). This phenotype is reminiscent of the reduced number of GABA-positive PC and MLF neurons reported in the cyclops mutant, which lacks the floor plate (Patel et al. 1994). Interestingly, the reduced Six3 expression levels in smu mutants are confined to the pretectum, while Six3 expression in the mesencephalic portion of the ventrocaudal cluster is down-regulated in the noi mutant (Tallafuss et al. 2003). This differential response of neurons in the ventrocaudal cluster to defects in Shh or Fgf8 signalling is a further indication that the MLF may originate from two distinct populations of neurons.

To gain further insight into the role of Shh signalling in early brain morphogenesis, we analysed the morphology of the initial tracts following ectopic expression of Shh in the rostral brain. In line with earlier studies (Watanabe \& Nakamura, 2000; Agarwala et al. 2001), we found that ectopic Shh caused a macroscopic phenotype. In particular, the border between mesencephalon and diencephalon was less distinct, indicating a change of tectum and pretectum morphology (not shown). Staining the axon tracts with an anti-neurofilament antibody revealed prominent alterations to the MLF (Fig. 3). Compared with control embryos, the $\mathrm{nMLF}$ appeared elongated, pointing to a possible role of Shh signalling in the specification of MLF neurons (Fig. 3C). In the mesencephalon, MLF axons diverted from the normal route just lateral to the floor plate, to traverse further dorsally, sometimes even projecting into the tectum (Fig. 3B,C). These pathfinding errors suggest a direct or indirect role of Shh in axon guidance for the MLF.

\section{Organizing the tegmentum: transcription factors mark different domains}

The specification of neuronal identity has been studied in detail in the spinal cord (Briscoe et al. 2000). Progenitor cells in the ventral neural tube express specific homeobox genes in response to a gradient of Shh signalling from the floor plate. The combinatorial expression of homeobox genes in the progenitor cells determines the expression of a second set of homeobox genes in the differentiating neurons, and thus establishes the types of neurons formed at a specific position along the dorsoventral axis. Along the whole rostrocaudal axis of the spinal cord, the homeobox genes expression domains form longitudinal columns, corresponding to the columns of neurons. Similar longitudinal 
CA $\beta$-GFP

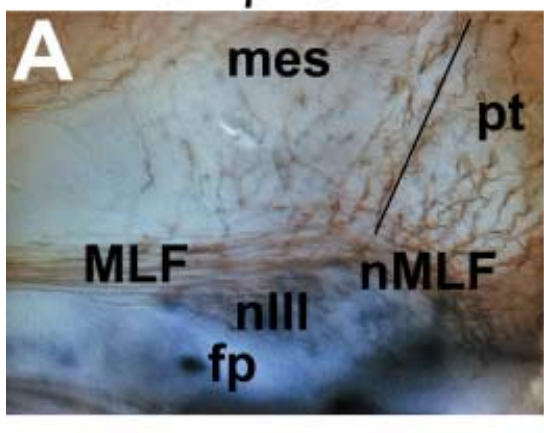

XeX-Shh

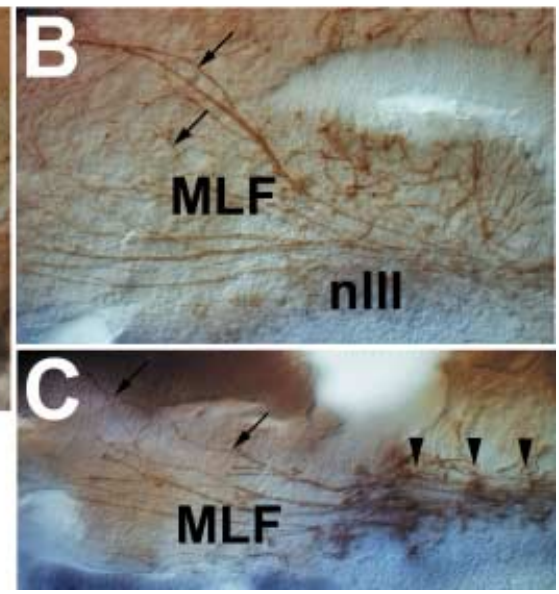

Fig. 3 Effects of ectopic SHH on the MLF. (A) The ventral midbrain-forebrain border region of an HH18 chick embryo electroporated with the CA $\beta$-GFP control construct, stained for Neurofilament-M. The organization of the nMLF and the course of the MLF axons are indistinguishable from wild-type embryos. (B,C) Phenotype of the MLF stained by anti-neurofilament antibody in HH18 (B) and HH16 (C) embryos electroporated with the pXEX-Shh construct (Agarwala et al. 2001). MLF axons are no longer constricted to ventral course along the floor plate. Instead, many of the axons grow into dorsal midbrain territory (arrows). In addition, the mesencephalic and pretectal subpopulations of the nMLF are no longer distinct, and the nMLF appears extended rostrally (arrowheads). fp, floor plate; mes, mesencephalon; MLF, medial longitudinal fascicle; nIII, oculomotor nucleus; $\mathrm{nMLF}$, nucleus of the MLF; pt, pretectum.

expression domains of homeobox genes have been described in the tegmentum of the mesencephalon and pretectum (Schubert et al. 1995; Sanders et al. 2002), where they underlie the proposed arc model of organizing the ventral midbrain (Sanders et al. 2002).

We have analysed the expression of Sax1 (Spann et al. 1994), Six3 (Bovolenta et al. 1998), Emx2 (Bell et al. 2001), Pax6 (Goulding et al. 1993) and Nkx2.2 (Ericson et al. 1997) in the developing chick tegmentum. Some of the expression patterns in the ventral midbrain have been reported previously (Schubert \& Lumsden, 2005). We have expanded the analysis to include $N k x 2.2$ as an additional marker, and also to report on the expression in the pretectum (Fig. 4). In the midbrain at $\mathrm{HH} 25$, the expression of all genes analysed is restricted to the tegmentum (Fig. 4A). Similar to the pattern in mice (Schubert et al. 1995), chick Pax6 is expressed in a single, longitudinal stripe stretching from the isthmus to the MFB (Fig. 4A5). This stripe has been described as separating arcs 2 and 3 in the arcuate model (Agarwala et al. 2001; Sanders et al. 2002). The Pax6 expression domain is located dorsal to the oculomotor nucleus (marked by $I s / 1$ expression), leaving a small gap (Fig. 4C5, 4D5). The gap between the $I s / 1$ and Pax6 expression domains is filled by the ventral stripes of Six 3 and $E m \times 2$ expression. Both genes are also expressed in a further stripe dorsal to the Pax 6 domain at this stage (arrows in Fig. 4B2, 4B3). The Sax1 expression domain does not reach as far caudally as those of Pax6,Emx2 and Six3, but overlaps with the single stripe of Pax6, and with the two stripes of $E m \times 2$ and Six3 close to the MFB (Fig. 4A1, 4B1). In addition, at the MFB the Sax1 signals extend further ventrally compared with the other three genes. In crosssections, the expression of Sax1, Six3, Emx2 and Pax6 can be seen to be restricted to the mantle layer of the mesencephalic tegmentum (Fig. 4C,D). In contrast, $N k x 2.2$ is expressed in the ventricular zone and mantle layer, in the latter overlapping with the expression of Pax6 and the dorsal domains of Emx2 and Six3 (Fig. 4C4, 4D4).

The expression of all genes analysed extends into the pretectum. However, the MFB is clearly visible as a discontinuity in the expression patterns. For Pax6, the mesencephalic signals peter out rostrally, while in the pretectum Pax6 is expressed strongly in a domain at the same dorsoventral level (Fig. 4B5, arrowhead). Emx2 and Six3 are still expressed in two stripes, but the dorsal stripe is much narrower than in the midbrain. The two stripes flank the Pax6 expression domain (arrowheads in Fig. 4B2, 4B3). In addition, Emx2 signals occupy a domain ventral to the two longitudinal stripes, corresponding to the red nucleus (Agarwala \& Ragsdale, 2002; black arrowhead in Fig. 4A2). The two Six3/Emx2 stripes merge into one longitudinal stripe at the rostral border of the pretectum, where the ventral expression of Pax 6 ceases. In contrast to $E m \times 2$ and Six3, the expression of Sax 1 in the pretectal tegmentum is restricted to a ventral domain, which extends from the ventral expression area near the MFB (Fig. 4A1, 4B1). The signals for $E m x 2$, Six3 and Sax1 traverse the ventral diencephalon up to the ZLI, where they turn dorsally to line the caudal border of the ZLI (Fig. 4F1-3). Nkx2.2 signals continue largely unchanged from the midbrain into the pretectum, overlapping with the Pax 6 domain and the adjacent areas (Fig. 4A4). 

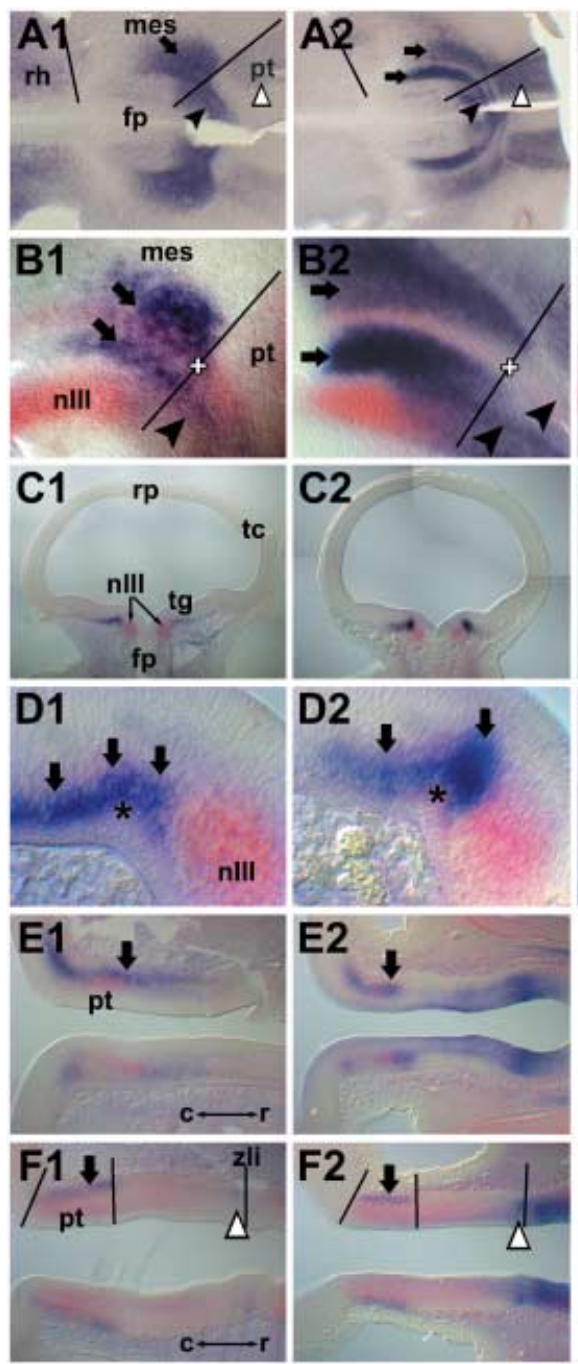

Sax1
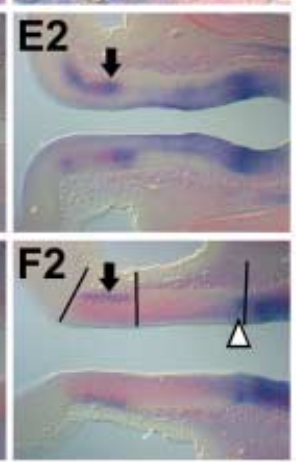

Emx2
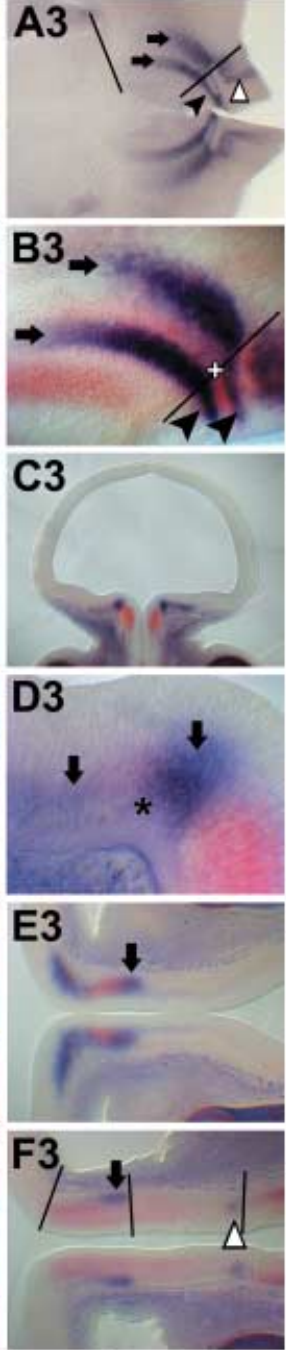

Six3
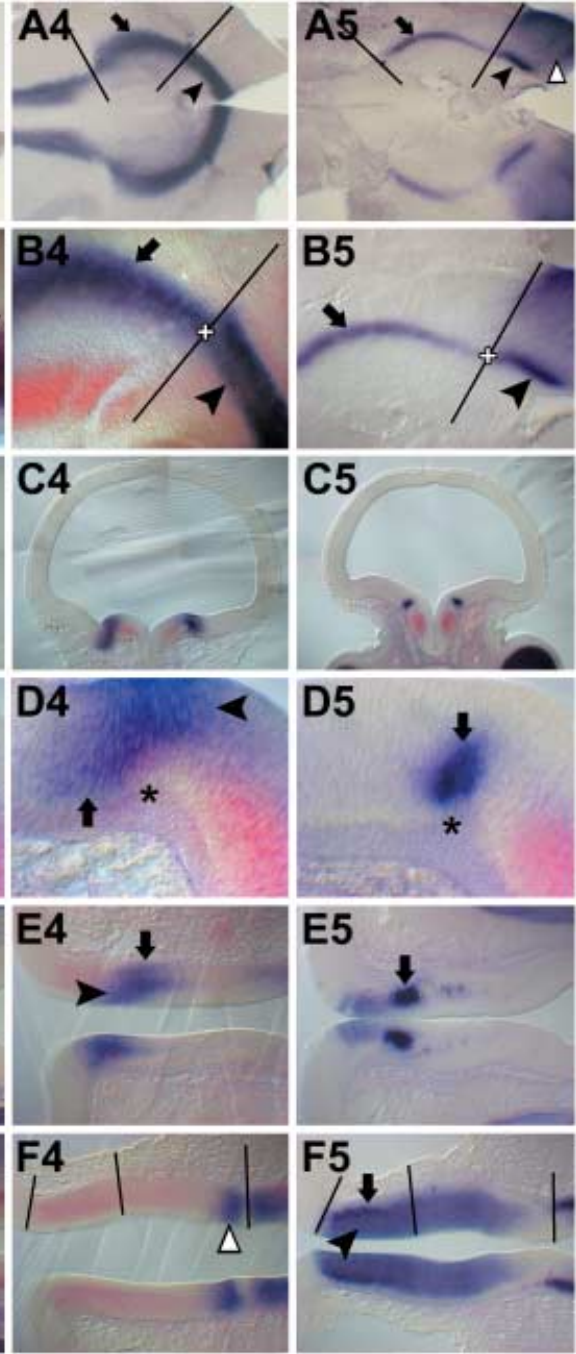

Nkx2.2

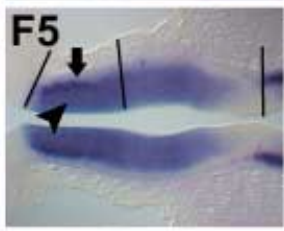

Pax6

Fig. 4 Expression patterns of homeobox genes in the tegmentum of early chick embryos. (A) Flat-mounted preparations of HH25 chick brains, stained by whole-mount in situ hybridization for the expression of Sax1 (A1), Em×2 (A2), Six3 (A3), Nkx2.2 (A4) and Pax6 (A5). (B) High magnification of the ventral midbrain-forebrain border region of HH25 embryos. B1-B3 and B5 are reproduced, with permission, from Schubert \& Lumsden (2005). Red staining indicates expression of Isl1 (ventrally, in the oculomotor nucleus) and Pax6. Sax1 is strongly expressed in the rostral ventral mesencephalon (arrow) and ventral pretectum (filled arrowhead), and weakly in the dorsal pretectum (open arrowhead). Emx2 and Six3 are expressed in two stripes in ventral mesencephalon (arrows) and pretectum (filled arrowheads). The stripes delineate the Pax6 expression domains in ventral mesencephalon (arrow) and pretectum (filled arrowhead). A ' + ' sign labels the position of the gap between mesencephalic and pretectal Pax6 signals in B1-5. Emx2, Six3 and Pax6 are also expressed in the dorsal pretectum (open arrowheads), where Six3 signals are largely confined to the rostral pretectum. Nkx2.2 signals form a ventral stripe extending from hindbrain into the diencephalon. (C) Coronal sections through the midbrain of HH2O chick embryos, with higher magnification of the tegmentum in D. Isl1 staining (red) in a broad superficial domain of the ventral tegmentum labels the oculomotor nucleus. Pax6 is expressed in a small group of superficial cells (arrow), dorsal to the nlll. The margin of the Pax6 signals is marked by an asterisk in D1-5. The signals for Sax1, Em×2 and Six3 are likewise confined to the mantle layer. Em×2 and Six3 are expressed ventral and dorsal to the Pax6 domain (arrows), while the Sax 1 domain encompasses the whole dorsoventral extent of the Emx2/Six3 and Pax6 domains (arrows). Sax 1 is confined to the most superficial cells, while the expression of Emx2, Six3 and Pax6 reaches closer to the ventricular zone. In contrast, Nkx2.2 is expressed in the ventricular zone (arrowhead) and the mantle layer (arrow) in an area covering the Pax 6 domain and cells just dorsal to it. (E,F) Coronal sections through the diencephalon of $\mathrm{HH} 20$ chick embryos, at the level of the ventral $(\mathrm{E})$ and dorsal (F) pretectum. The rostral-caudal axis is indicated (E1,F1). In the ventral pretectum, Pax6, Emx2, Six3 and Sax1 are expressed in the outer mantle layer (arrows), similar to the ventral mesencephalon. Nkx2.2 signals again span across the ventricular zone (filled arrowhead) and mantle layer (arrow). In the dorsal pretectum, Pax6 is expressed throughout the ventricular zone and mantle layer (filled arrowhead), with a marginal zone of stronger signals (arrow). The signals for Sax1, Em×2 and Six3 are confined to the outer mantle layer (arrows). Six3 expression is restricted to the rostral pretectum, while the expression of Sax 1 and Emx2 spans the whole dorsal pretectum. $\mathrm{Nkx2.2}$ is not expressed dorsally. In addition to the pretectum, signals for Sax1, Emx2, Six3 and Nkx2.2 (open arrowheads) outline the caudal margin of the zona limitans intrathalamica, an area depleted of Pax6 signals. c, caudal; fp, floor plate; mes, mesencephalon; nlll, oculomotor nucleus; pt, pretectum; r, rostral; rh, rhombencephalon; rp, roof plate; tc, tectum; ZLI, zona limitans intrathalamica. 
In contrast to their strictly basal expression in the midbrain, in the pretectum most of the genes analysed are expressed additionally in the alar plate (white triangles in Fig. 4A, arrows in Fig. 4F). Pax6 is expressed broadly in the whole dorsal pretectum (Fig. 4A5, 4F5). Indicating a role for Pax 6 in the fate specification of TPC neurons, the dorsal neurons of the TPC fail to develop in the Pax6 mutant Small eye (Stoykova et al. 1996; Mastick et al. 1997). On the other hand, Sax1, Six3 and Emx2 expression in the dorsal pretectum is limited to the outer mantle layer (Fig. 4A1-3, 4F1-3). Interestingly, forced expression of $E m \times 2$ under the control of the Otx2 promoter results in the loss of the TPC (Suda et al. 2001), although it is not clear whether this phenotype is due to an earlier patterning defect or to misspecification of neurons. Sax1 and Emx2 are expressed equally in rostral and caudal pretectum, while Six3 signals are mainly located in the rostral half of the pretectum.

\section{Specifying neuronal fate: Sax1 governs MLF development}

This differential expression of homeobox genes in the tegmentum may establish a molecular code for the specification of neuronal fate in the ventral brain (Schubert \& Lumsden, 2005). In line with this model, Emx2 is involved in the formation of the red nucleus (Agarwala \& Ragsdale, 2002), and several homeobox genes act in the development of dopaminergic neurons in the later midbrain (reviewed in Prakash \& Wurst, 2006). However, it is unclear whether homeobox genes also participate in the specification of ventral neurons contributing to the early axon scaffold. The expression of several homeobox genes in close proximity to the nMLF during the differentiation of the first MLF neurons suggests a possible role of these transcription factors in neuronal specification, similar to the spinal cord. In particular, the Sax genes in mouse (Schubert et al. 1995; Simon \& Lufkin, 2003), zebrafish (Bae et al. 2004) and chick (Schubert \& Lumsden, 2005) are expressed in the tegmentum, closely associated with the nMLF. Neurofilament staining (Fig. 5A) and retrograde labelling of the MLF (Fig. 5B) combined with in situ hybridization for Sax 1 mRNA reveal that Sax1 signals in the chick overlap the position of the nMLF (Schubert \& Lumsden, 2005). Interestingly, in chick the expression domain of Sax1 at the MFB overlaps with that of Six3 (Fig. 4), which in zebrafish marks the ventrocaudal cluster containing the nMLF (Tallafuss et al. 2003).

To investigate the function of Sax1 in the formation of the MLF, the MLF was analysed in chick embryos expressing Sax 1 ectopically in the midbrain (Fig. 5C-F; Schubert \& Lumsden, 2005). Neurofilament staining revealed alterations in neuronal morphology, affecting both tectum and tegmentum. In particular, while the MLF normally runs as a bundle close to the floor plate (Fig. 5C), in Sax1electroporated embryos the MLF was much wider, mirroring the dorsal expansion of the MLF following ectopic Shh expression (compare Figs 3B and 5D). The irregular course of the MLF was visible as soon as 1 day after electroporation (Fig. 3D), and the effect was even more pronounced after 2 days (Schubert \& Lumsden, 2005). Since ectopic Sax1 expression is induced by Shh (M. Ahsan and F. R. Schubert, unpublished data), it is likely that Sax 1 mediates some of the phenotype seen after ectopic Shh expression. To analyse the effect on MLF morphology further, we stained specifically the MLF by retrograde labelling from the rostral hindbrain (Fig. 5E,F). In control embryos, the mesencephalic and pretectal parts of the nMLF are distinct (Fig. 5E). The MLF neurons in the rostral mesencephalon are located more dorsally, and first extend their axons ventrally before turning caudally. The pretectal MLF neurons are found in a more ventral position, and their axons grow only caudally. In Sax1-electroporated embryos, the distinction between the two parts of the nMLF is lost. The number of MLF neurons is increased, and the majority of these neurons extend their axons just caudally (Fig. 5F).

The distinctive expression patterns of homeobox genes in the tegmentum suggest that their expression is precisely controlled. In the spinal cord, homeobox gene expression along the dorsoventral axis is initially controlled by a differential response to graded Shh signalling, followed by cross-repressive interactions between specific homeobox genes to sharpen the expression boundaries (Briscoe et al. 2000). Shh can likewise induce the expression of different homeobox genes in the tegmentum in a dose-dependent manner (Agarwala et al. 2001). To investigate whether the distinct expression patterns of homeobox genes in the tegmentum might also be shaped by reciprocal transcriptional regulation, the expression pattern of $E m \times 2$ was studied after ectopic Sax1 expression (Fig. 5G,H; Schubert \& Lumsden, 2005). While control-electroporated embryos showed the normal ventral signals of $E m \times 2$ in mesencephalon and pretectum (Fig. 5G), ectopic Sax1 suppressed Emx2 expression (Fig. 5H). In addition, a VP16-Sax1 construct induced ectopic Emx2 expression cell-autonomously (Schubert \& Lumsden, 2005), arguing for a direct regulation of Emx2 expression by Sax1. Interestingly, ectopic Emx2 also affects the expression of Sax1 (M. Ahsan and F. R. Schubert, unpublished data), indicating that, similar to the spinal cord (Briscoe et al. 2000), the transcription factors in ventral mesencephalon and pretectum regulate each other. However, it should be noted that the crossrepressive interactions in the spinal cord regulate gene expression in the progenitor cells, while Sax1 and Emx2 are expressed in the mantle layer, presumably by differentiating neurons.

\section{Pathfinding: axon guidance molecules delineate the course of the MLF}

The fate specification of neurons is the first step in establishing the MLF, and is quickly followed by the outgrowth 

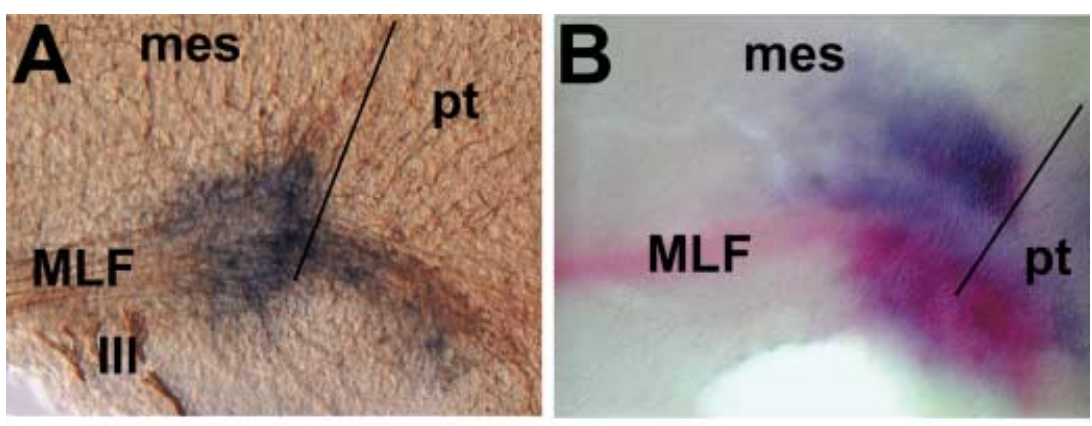

\section{CA $\beta$-GFP}
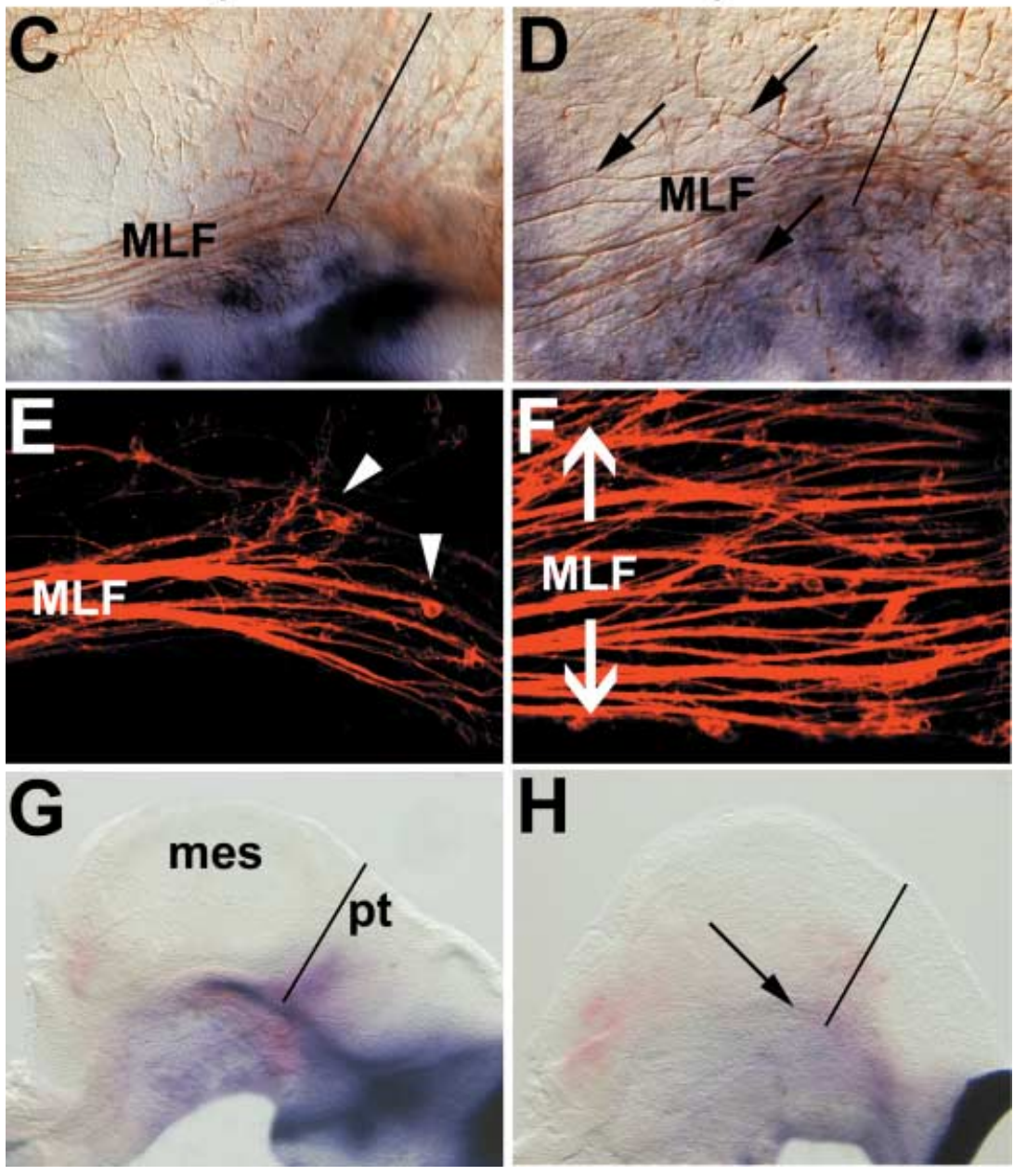

H
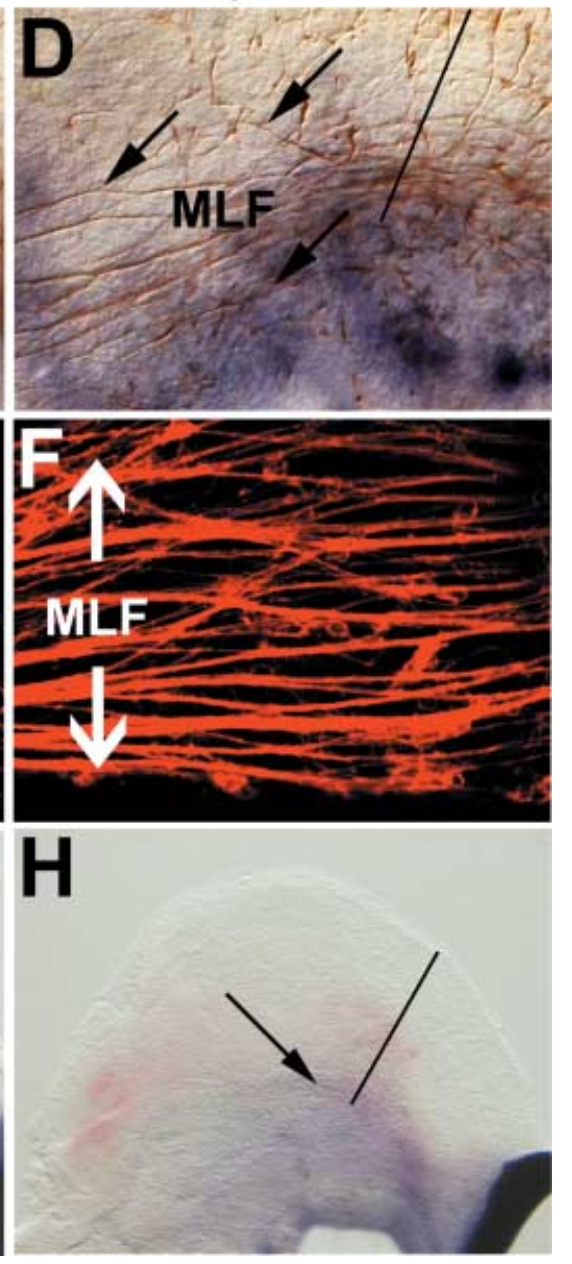

Fig. 5 Sax1 function in the formation of the MLF. (A) Ventral midbrain-forebrain border region of an $\mathrm{HH} 19$ chick embryo, stained for Sax1 mRNA (blue) and Neurofilament-M protein (brown). The Sax1 signals overlay the mesencephalic and pretectal parts of the nMLF. (B) Retrograde labelling of the MLF (red), combined with staining for Sax1 expression (blue) in an $\mathrm{HH} 23$ embryo. The MLF can be traced back to the Sax1 expression domain. (C-F) Effect of ectopic Sax1 expression on the formation of the MLF. Staining with anti-neurofilament antibody (C) or retrograde labelling (E) shows the normal appearance of the MLF in control-electrporated embryos at HH18. In contrast, ectopic Sax1 leads to erroneous path ( $D$, arrows) and enlargement (F) of the MLF. $(\mathrm{G}, \mathrm{H})$ Interaction of Sax1 with Emx2. At HH18, Emx2 is expressed in a single ventral stripe in wild-type (not shown) and control-electroporated ( $G$ ) embryos. The mesencephalic and diencephalic expression domains of $E m \times 2$ are lost in embryos expressing ectopic Sax1 (H). All images are reproduced, with permission, from Schubert \& Lumsden (2005). III, oculomotor nerve; mes, mesencephalon; MLF, medial longitudinal fascicle; pt, pretectum; TPC, tract of the posterior commissure. of axons. The MLF axons grow strictly caudally, in a loosely fasciculated bundle close to the ventral midline. The three main decisions for MLF axons likely to be controlled by axon guidance molecules are (1) to grow caudally not rostrally, (2) to stay ipsilateral rather than to cross the midline, and (3) to grow close to the midline not dorsally. Several members of the established families of axon guidance molecules are expressed in the tegmentum during MLF formation, but a detailed study of their expression patterns in correlation to axon outgrowth is lacking. To gain further insight into the possible axon guidance mechanisms in the formation of the MLF, we have examined the expression of class 3 Semaphorins, Netrins, Slits and their respective receptors during the initial stages of MLF development in the chick embryo (K. Riley and F. R. Schubert, unpublished data). Our preliminary data indicate that Sema3A is expressed just rostral to the nMLF, and that Sema3A signalling, mediated by Neuropilin1, contributes to preventing MLF growth into the forebrain. In zebrafish, a similar function can be attributed to a different Semaphorin: Sema3D is expressed just rostral to the ventrocaudal cluster, and the knock-down of Sema3D function by morpholinos allows MLF axons to grow into the forebrain territory (Wolman et al. 2004). 
Possible candidates for controlling MLF growth along the floor plate are Netrins and Shh, which has recently been shown to act as a guidance molecule for longitudinal axon growth in the spinal cord (Bourikas et al. 2005). The dorsal expansion of the MLF following ectopic Shh expression and the misprojection of MLF axons towards the tectum suggest that the guidance of MLF axons is affected by Shh. In the zebrafish floor plate mutant cyclops, MLF axon outgrowth is also disturbed (Patel et al. 1994). However, while these data demonstrate the importance of floor plate signals for the MLF, it is not clear whether the guidance effects can be directly attributed to Shh, or whether the expression of other guidance molecules like Netrins is affected by Shh signalling. In zebrafish, the Netrin receptor DCC is expressed in the ventrocaudal cluster (Hjorth et al. 2001), suggesting that Netrin might function as an attractive guidance molecule for MLF axons.

The expression pattern of the Slit ligands is not immediately suggestive of a guidance function during the course of the MLF axons across the mesencephalon. However, the broad bundle of MLF axons becomes focused at the isthmus, and repulsion by Slits expressed at the isthmus has been attributed to this narrowing of the tract (Molle et al. 2004). Another possible function for Slits might be to prevent MLF axons from crossing the ventral midline.

\section{Concluding remarks}

The organization of the vertebrate brain is outlined initially by an early scaffold of longitudinal and transversal axon tracts. Prominent among these is the MLF, which in many vertebrates is the first tract to be established in the brain. The MLF neurons are located at the ventral midbrainforebrain border, where they form two distinct populations: scattered neurons in the mesencephalon, dorsal to the path of the MLF, and a group of densely packed neurons in the pretectum, under the path of the MLF. Surprisingly little is known about the possible role and later fate of these two populations, though the early MLF neurons are often described as developing into the rostral interstitial nucleus of Cajal. This notion is backed by retrograde labelling studies in young rat embryos, where MLF axons were traced back to the nucleus (Auclair et al. 1993). Despite our patchy knowledge of early MLF anatomy, we are beginning to understand how patterning molecules, transcription factors and axon guidance molecules govern the formation of this early tract.

The analysis of mutant zebrafish has suggested that patterning by Fgf8 and Shh signalling could influence the formation of the ventrocaudal cluster of neurons, which includes the nucleus of the MLF (Tallafuss et al. 2003). In this study, we have shown that ectopic Shh in the embryonic chick brain causes an expansion of the nucleus of the $M L F$, and results in the aberrant pathway of MLF axons. These results argue for a dual role of Shh in the formation of the MLF, both in fate specification and in axon guidance.
Likely downstream effectors of Shh signalling in the fate specification of MLF neurons are homeobox genes that are expressed in the tegmentum of the mesencephalon and pretectum. In particular, the expression of Sax 1 correlates with the position of the MLF neurons in the mesencephalon and pretectum, and ectopic expression of Sax 1 causes an expansion of the MLF (Schubert \& Lumsden, 2005). Patterning and fate specification in the tegmentum might follow a similar mechanism to that demonstrated in the spinal cord (Briscoe et al. 2000). Several homeobox genes are expressed at distinct dorsoventral positions in the tegmentum (Sanders et al. 2002; Schubert \& Lumsden, 2005), and some of these transcription factors, such as Sax 1 and $E m \times 2$, reciprocally regulate each other (Schubert \& Lumsden, 2005).

The MLF is the main longitudinal tract in the early embryo, and its axons follow a strictly caudal course. MLF axons therefore have to be prevented from entering the forebrain, and studies in zebrafish (Wolman et al. 2004) and chick (our unpublished data) have shown that class III Semaphorins in the ventral diencephalon, mediated by Neuropilin1, repel MLF axons (Fig. 6B). On their path through the mesencephalon, MLF axons remain ventral, suggesting a possible attraction by the floor plate. In line with this hypothesis, we have shown that ectopic expression of Shh results in dorsal misrouting of MLF axons. This result suggests that Shh itself, or other molecules downstream of Shh signalling, like Netrins, are involved in the attraction of MLF axons to the floor plate region (Fig. 6B). Finally, Slit signalling at the midbrain-hindbrain border has been shown to focus MLF axons to a narrow channel (Molle et al. 2004).

The MLF has a simple organization, it pioneers the ventral longitudinal tract in the early embryo, and it is conserved through all vertebrate classes investigated. These features make it an ideal model to understand fully the formation of a complete axon tract, including the differentiation and specification of its neurons, the outgrowth of its axons and the recognition of its targets. We have made progress in unravelling the molecular events that regulate the formation of the MLF, but several questions remain unanswered. How different are the morphologically distinguishable two subpopulations of MLF neurons? Are they specified by different molecular mechanisms? What are the roles of genes like Emx2, Six3 or Pax6 in fate specification in the tegmentum? Which are the axon guidance molecules attracting the MLF to the floor plate? Are other guidance molecules involved in repelling the MLF from the alar plate, or preventing it from crossing the floor plate? What are the targets of the first MLF neurons? Future studies, combining the anatomical and molecular characterization of the MLF with the genetic dissection of regulatory pathways, will unravel the molecular mechanisms that govern the formation of this pioneering longitudinal axon tract. 


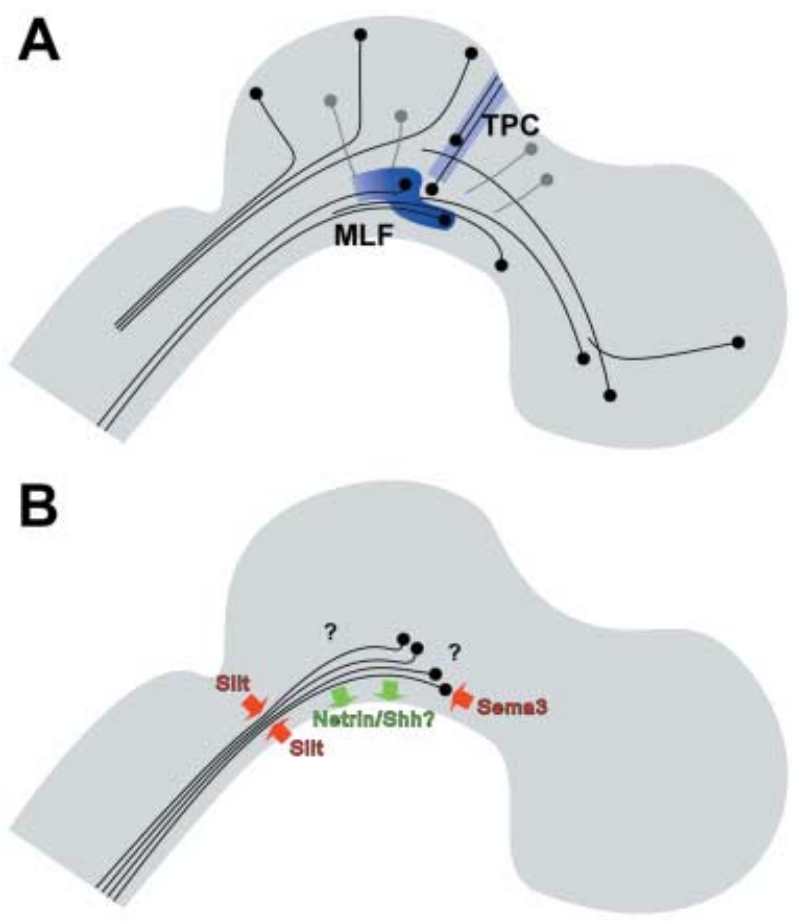

Fig. 6 Fate specification and axon guidance of the MLF. (A) Schematic representation of Sax 1 expression (blue) mapped onto the early axon tracts. Sax 1 expression in the tegmentum of mesencephalon and pretectum correlates with the position of the $\mathrm{nMLF}$, and the phenotype of Sax1 electroporations implies Sax 1 in the specification of MLF neuron fate. (B) Known and proposed axon guidance molecules that influence MLF growth. MLF neurons are represented in black, attractive signals in green, and repellent signals in red. Sema3D (zebrafish, Wolman et al. 2004) or Sema3A (in chick, our unpublished data) prevent MLF axons from growing rostrally. Netrin and/or Shh are likely candidates to attract MLF axons to its course close to the floor plate: ectopic Shh causes misrouting of the MLF (this study), while the Netrin receptor DCC is expressed in the nMLF (Hjorth et al. 2001). The repellents Slit1 and Slit2 are expressed in ventral and dorsal isthmus, focusing the MLF axons into a narrow channel (Molle et al. 2004). It is unclear whether other factors might be required to prevent MLF axons from growing into the alar plate of pretectum or mesencephalon. MLF, medial longitudinal fascicle; TPC, tract of the posterior commissure.

\section{Acknowledgements}

We are indebted to A. Lumsden for his crucial support during the initial phase of the project. We would like to thank $C$. Ragsdale for the pXeX-Shh construct, J. Gilthorpe and A. Hunter for the pCA $\beta$ LINK-IRESeGFPm 5 construct, and A. Fainsod, P. Gruss and T. Jessell for providing probes for in situ hybridization. A special thanks to the Company of Biologists for permission to reproduce figures from our recent publication. We gratefully acknowledge the support of the Royal Society and the BBSRC for our studies on neuronal specification and axon guidance. K.R. is supported by an IBBS bursary from the University of Portsmouth.

\section{References}

Agarwala S, Sanders TA, Ragsdale CW (2001) Sonic hedgehog control of size and shape in midbrain pattern formation. Science 291, 2147-2150.
Agarwala S, Ragsdale CW (2002) A role for midbrain arcs in nucleogenesis. Development 129, 5779-5788.

Auclair F, Belanger MC, Marchand R (1993) Ontogenetic study of early brain stem projections to the spinal cord in the rat. Brain Res Bull 30, 281-289.

Bae YK, Shimizu T, Muraoka O, et al. (2004) Expression of sax1/nkx1.2 and sax2/nkx1.1 in zebrafish. Gene Expr Patterns 4, 481-486.

Bell E, Ensini M, Gulisano M, Lumsden A (2001) Dynamic domains of gene expression in the early avian forebrain. Dev Biol 236, 76-88.

Bourikas D, Pekarik V, Baeriswyl T, et al. (2005) Sonic hedgehog guides commissural axons along the longitudinal axis of the spinal cord. Nat Neurosci 8, 297-304.

Bovolenta P, Mallamaci A, Puelles L, Boncinelli E (1998) Expression pattern of cSix3, a member of the Six/sine oculis family of transcription factors. Mech Dev 70, 201-203.

Briscoe J, Pierani A, Jessell TM, Ericson J (2000) A homeodomain protein code specifies progenitor cell identity and neuronal fate in the ventral neural tube. Cell 101, 435-445.

Chapman SC, Schubert FR, Schoenwolf GC, Lumsden A (2002) Analysis of spatial and temporal gene expression patterns in blastula and gastrula stage chick embryos. Dev Biol 245, 187-199.

Chedotal A, Pourquie O, Sotelo C (1995) Initial tract formation in the brain of the chick embryo: selective expression of the BEN/ SC1/DM-GRASP cell adhesion molecule. Eur J Neurosci 7, 198212.

Chitnis AB, Kuwada JY (1990) Axonogenesis in the brain of zebrafish embryos. J Neurosci 10, 1892-1905.

Doldan MJ, Prego B, Holmqvist B, Helvik JV, de Miguel E (2000) Emergence of axonal tracts in the developing brain of the turbot (Psetta maxima). Brain Behav Evol 56, 300-309.

Easter SS Jr, Ross LS, Frankfurter A (1993) Initial tract formation in the mouse brain. J Neurosci 13, 285-299.

Ericson J, Rashbass P, Schedl A, et al. (1997) Pax6 controls progenitor cell identity and neuronal fate in response to graded Shh signaling. Cell 90, 169-180.

Goulding MD, Lumsden A, Gruss P (1993) Signals from the notochord and floor plate regulate the region-specific expression of two Pax genes in the developing spinal cord. Development 117, 1001-1016.

Hamburger V, Hamilton HL (1951) A series of normal stages in the development of the chick embryo. J Morph 88, 49-92.

Hartenstein V (1993) Early pattern of neuronal differentiation in the Xenopus embryonic brainstem and spinal cord. J Comp Neurol 328, 213-231.

Hjorth JT, Gad J, Cooper H, Key B (2001) A zebrafish homologue of deleted in colorectal cancer (zdcc) is expressed in the first neuronal clusters of the developing brain. Mech Dev 109, 105109.

Hjorth J, Key B (2002) Development of axon pathways in the zebrafish central nervous system. Int J Dev Bio/ 46, 609-619.

Kiecker C, Lumsden A (2004) Hedgehog signaling from the ZLI regulates diencephalic regional identity. Nat Neurosci 7, 12421249.

Macdonald R, Scholes J, Strahle U, et al. (1997) The Pax protein Noi is required for commissural axon pathway formation in the rostral forebrain. Development 124, 2397-2408.

Mastick GS, Easter SS Jr (1996) Initial organization of neurons and tracts in the embryonic mouse fore- and midbrain. Dev Biol 173, 79-94.

Mastick GS, Davis NM, Andrew GL, Easter SS Jr (1997) Pax-6 functions in boundary formation and axon guidance in the embryonic mouse forebrain. Development 124, 1985-1997. 
McConnell JA, Sechrist JW (1980) Identification of early neurons in the brainstem and spinal cord. I. An autoradiographic study in the chick. J Comp Neurol 192, 769-783.

Molle KD, Chedotal A, Rao Y, Lumsden A, Wizenmann A (2004) Local inhibition guides the trajectory of early longitudinal tracts in the developing chick brain. Mech Dev 121, 143-156.

Nakamura H, Watanabe Y (2005) Isthmus organizer and regionalization of the mesencephalon and metencephalon. Int J Dev Biol 49, 231-235.

Nieuwenhuys R (1998) Development of fibre systems. The Central Nervous System of Vertebrates, Vol. 1 (eds Nieuwenhuys R, ten Donkelaar HJ, Nicholson C), pp. 256-271. Berlin: Springer-Verlag.

Patel CK, Rodriguez LC, Kuwada JY (1994) Axonal outgrowth within the abnormal scaffold of brain tracts in a zebrafish mutant. J Neurobio/ 25, 345-360.

Prakash N, Wurst W (2006) Genetic networks controlling the development of midbrain dopaminergic neurons. J Physio/ 575, 403-410.

Puelles L, Amat JA, Martinez-de-la-Torre M (1987) Segmentrelated, mosaic neurogenetic pattern in the forebrain and mesencephalon of early chick embryos. I. Topography of AChEpositive neuroblasts up to stage HH18. J Comp Neuro/ 266, 247268.

Ross LS, Parrett T, Easter SS Jr (1992) Axonogenesis and morphogenesis in the embryonic zebrafish brain. J Neurosci 12, 467482.

Sanders TA, Lumsden A, Ragsdale CW (2002) Arcuate plan of chick midbrain development. J Neurosci 22, 10742-10750.

Schubert FR, Fainsod A, Gruenbaum Y, Gruss P (1995) Expression of the novel murine homeobox gene Sax-1 in the developing nervous system. Mech Dev 51, 99-114.

Schubert FR, Lumsden A (2005) Transcriptional control of early tract formation in the embryonic chick midbrain. Development 132, 1785-1793.

Shanmugalingam S, Houart C, Picker A, et al. (2000) Ace/Fgf8 is required for forebrain commissure formation and patterning of the telencephalon. Development 127, 2549-2561.
Simon R, Lufkin T (2003) Postnatal lethality in mice lacking the Sax2 homeobox gene homologous to Drosophila S59/slouch: evidence for positive and negative autoregulation. Mol Cell Biol 23, 9046-9060.

Skene JH, Willard M (1981) Axonally transported proteins associated with axon growth in rabbit central and peripheral nervous systems. J Cell Biol 89, 96-103.

Spann P, Ginsburg M, Rangini Z, Fainsod A, Eyal-Giladi H, Gruenbaum $Y$ (1994) The spatial and temporal dynamics of Sax1 (CHox3) homeobox gene expression in the chick's spinal cord. Development 120, 1817-1828.

Stoykova A, Fritsch R, Walther C, Gruss P (1996) Forebrain patterning defects in Small eye mutant mice. Development 122, 3453-3465.

Suda Y, Hossain ZM, Kobayashi C, et al. (2001) Emx2 directs the development of diencephalon in cooperation with Otx2. Development 128, 2433-2450.

Tallafuss A, Adolf B, Bally-Cuif L (2003) Selective control of neuronal cluster size at the forebrain/midbrain boundary by signaling from the prechordal plate. Dev Dyn 227, 524-535.

Tsuchida T, Ensini M, Morton SB, et al. (1994) Topographic organization of embryonic motor neurons defined by expression of LIM homeobox genes. Cell 79, 957-970.

Varga ZM, Amores A, Lewis KE, et al. (2001) Zebrafish smoothened functions in ventral neural tube specification and axon tract formation. Development 128, 3497-3509.

Watanabe Y, Nakamura H (2000) Control of chick tectum territory along dorsoventral axis by Sonic hedgehog. Development 127, 1131-1140.

Wilson SW, Ross LS, Parrett T, Easter SS Jr (1990) The development of a simple scaffold of axon tracts in the brain of the embryonic zebrafish, Brachydanio rerio. Development 108, 121-145.

Windle WF, Austin MF (1936) Neurofibrillar development in the central nervous system of chick embryos up to 5 days' incubation. J Comp Neurol 63, 431-463.

Wolman MA, Liu Y, Tawarayama H, Shoji W, Halloran MC (2004) Repulsion and attraction of axons by semaphorin3D are mediated by different neuropilins in vivo. J Neurosci 24, 8428-8435. 